\title{
Produção científica da residência em gerência dos serviços de enfermagem de 2006 a 2011
}

\section{Scientific production of residence in the management of nursing services 20062011}

\author{
Camila Severi Zanoni ${ }^{1}$; Maria do Carmo Lourenço Haddad²; Marli Terezinha \\ Oliveira Vannuchi ${ }^{3}$; Larissa Gutierrez de Carvalho Silva ${ }^{4}$; Mara Solange Gomes \\ Dellaroza $^{5}$; Mariana Angela Rossaneis ${ }^{6}$
}

\begin{abstract}
Resumo
Estudo retrospectivo, descritivo, documental e quantitativo realizado para descrever a produção científica da Residência de Gerência dos Serviços de Enfermagem do Hospital Universitário da Universidade Estadual de Londrina, de 2006 a 2011. As informações foram coletadas por instrumento que identificou título, autores, periódico e classificação no QUALIS/Coordenação de Aperfeiçoamento de Pessoal de Nível Superior dos artigos produzidos. Foram analisados 69 artigos, sendo 43,5\% publicados, 31,9\% submetidos a análise do corpo editorial, $4,3 \%$ no prelo, $14,5 \%$ resubmetidos após recusa na $1^{\mathrm{a}}$ submissão e 5,8\% aguardam submissão. Houve predomínio da submissão a periódicos B1 (44,6\%). Dos 96 trabalhos, 15 foram apresentados em eventos regionais, $60 \mathrm{em}$ nacionais e $21 \mathrm{em}$ internacionais, sendo 93,7\% como pôster e nove premiados. A complexidade do campo da saúde e as exigências do mercado de trabalho mostram a importância da pesquisa em Enfermagem desenvolvida durante a Residência como possibilitadora da reflexão e transformação da prática profissional.
\end{abstract}

Palavras-chave: Enfermagem. Pesquisa. Educação de Pós-Graduação em enfermagem.

\begin{abstract}
Retrospective, descriptive, documentary and quantitative study conducted to describe the scientific production of the Housing Management Services Nursing, University Hospital of Londrina State University, from 2006 to 2011. Information was collected by an instrument that identified the title, author, journal and classification in QUALIS / Coordination of Improvement of Higher Education Personnel of the articles produced. We analyzed 69 articles, $43.5 \%$ were published, $31.9 \%$ underwent the editorial board, $4.3 \%$ in the press, $14.5 \%$ resubmitted after rejection in the 1 st submission and $5.8 \%$ are awaiting submission. There was a predominance of submission to journals B1 (44.6\%). Of the 96 studies, 15 were presented at regional events in 60 national and 21 international, and $93.7 \%$ as a poster and nine received awards. The complexity of the health field and the demands of the labor market show the importance of research in nursing developed during the residency as enabler of reflection and transformation of professional practice.
\end{abstract}

Keywords: Nursing. Research. Graduate Nursing Education.

\footnotetext{
${ }^{1}$ Enfermeira. Especialista em Gerência dos Serviços de Enfermagem pela Universidade Estadual de Londrina.

${ }^{2}$ Enfermeira. Doutora em Enfermagem. Docente do departamento de Enfermagem da Universidade Estadual de Londrina.

${ }^{3}$ Enfermeira. Doutora em Enfermagem. Docente do departamento de Enfermagem da Universidade Estadual de Londrina.

${ }^{4}$ Enfermeira. Doutoranda em Enfermagem na Escola de Enfermagem de Ribeirão Preto. Docente do departamento de Enfermagem da Universidade Estadual de Londrina.

${ }^{5}$ Enfermeira. Doutora em Enfermagem. Docente do departamento de Enfermagem da Universidade Estadual de Londrina.

${ }^{6}$ Enfermeira. Mestre em Ciências. Doutoranda em Enfermagem na Universidade Estadual de Maringá.
} 


\section{Introdução}

A pesquisa, como atividade que possibilita a reflexão e a transformação da prática profissional, constitui uma ferramenta importante e indispensável no processo de trabalho do enfermeiro (SILVA et al, 2009).

A enfermagem, para acompanhar a evolução técnico-científica vigente na era da informação e exercer com qualidade o seu papel social, necessita cada vez mais do posicionamento de profissionais comprometidos com a transformação social e profissional (GUARIENTE et al., 2010).

Esse cenário mostra a necessidade persistente de melhorar a qualificação dos profissionais de enfermagem em todos os níveis de formação, inclusive de pós-graduação, e de fortalecer a articulação entre o cuidado de enfermagem e o gerenciamento do cuidado, sobretudo, por parte dos enfermeiros (MUNARI et al., 2011).

$\mathrm{Na}$ busca de estratégias para transformar o fazer, a enfermagem investe nos cursos de pósgraduação por estes representarem recursos que poderão proporcionar qualidade, credibilidade e eficácia no desempenho de sua prática profissional A realização de curso de pós-graduação demostra o compromisso dos enfermeiros com a qualidade de seu desempenho profissional, o que é favorável para o alcance de bons resultados na sua atuação (SANTOS; CASTRO, 2010).

Considerando as exigências do mercado de trabalho quanto à necessidade de capacitação do profissional graduado, o Departamento de Enfermagem daUniversidade Estadual de LondrinaPR em parceria com a Diretoria de Enfermagem do Hospital Universitário de Londrina (HUL) implantou em 2006 o Programa de Residência em Enfermagem em cinco especialidades, dentre as quais se discutirá particularmente a residência em Gerência dos Serviços de Enfermagem.

A Residência caracteriza-se como treinamento em serviço e possibilita ao enfermeiro recém- graduado o crescimento profissional através do aperfeiçoamento de habilidades técnicas a obtenção do título de especialista na área de escolha. Este programa, com carga horária de 5.760 horas distribuídas em dois anos, está estruturado conforme a Resolução 259/2001 do Conselho Federal de Enfermagem (COFEN), que regulamenta os cursos de pós-graduação Latu Sensu (CONSELHO FEDERAL DE ENFERMAGEM, 2001), e tem como requisito obrigatório para obtenção do certificado de conclusão de curso o desenvolvimento de, no mínimo, dois artigos científicos por aluno associado a apresentações de trabalhos em eventos locais e nacionais.

A carga horária do primeiro ano inclui conteúdos teóricos (375 horas) que são desenvolvidos em comum a todas as modalidades ofertadas no Programa de Residência em Enfermagem. Inclui também conteúdos específicos (630 horas) da área da gerência, ministrados sob a forma de aulas dialogadas, seminários, estudos de caso, e outras.

As atividades práticas da Residência são realizadas nas unidades de urgência/emergência, de internação e de terapia intensiva de adultos em hospitais de níveis secundário e terciário da cidade de Londrina-PR. A carga horária prática do $1^{\mathrm{o}}$ ano é de 1.875 horas e no $2^{\circ}$ ano de 2.880 horas desenvolvidas em jornada de 40 horas semanais, incluindo um plantão de 12 horas nos finais de semana. Já as atividades teóricas totalizam 20 horas semanais.

No segundo ano, os estágios ocorrem em serviços da alta gerência de enfermagem do HUL, na Santa Casa de Londrina e na Divisão de Assistência à Saúde da Comunidade da Universidade Estadual de Londrina.

Durante todo o curso, o aluno é avaliado de forma sistemática e individualmente, por meio do portfólio reflexivo, das reuniões mensais entre residentes, docentes e enfermeiros dos campos de estágio, e da oficina de trabalho realizada anualmente para avaliar o desenvolvimento 
da residência no respectivo ano e planejar as atividades para o ano seguinte.

As investigações são realizadas na linha de pesquisa dos docentes que são: a formação de recursos humanos em enfermagem, gestão de serviços de enfermagem, saúde do trabalhador, psicodinâmica do trabalho em saúde, gerenciamento de risco, qualidade da assistência de enfermagem e o cuidado de enfermagem nas fases do ciclo vital e nos diversos níveis assistenciais.

Ressalta-se a importância de se definir as linhas de pesquisa, que se prende as exigências atuais no processo de produção do conhecimento e se tornou um empreendimento coletivo, realizado de modo progressivo e contínuo ao longo do tempo (OLIVEIRA; HADDAD; BORTOLETTO, 2010).

A prática em serviço realizada por estudantes em programas de pós graduação Latu sensu, tem papel fundamental na construção de gestores, em meio à complexidade dos sistemas de saúde, sendo de fundamental importância na construção de identidades profissionais e na produção do cuidado, considerando a busca por qualidade, integralidade, eficiência e controle de custos nos sistemas de saúde.

Muitas são as vantagens de um curso de Residência planejado e bem estruturado, além do preparo técnico científico, o residente adquire segurança profissional para o desenvolvimento das atividades práticas, se conscientiza da necessidade do aprendizado complementar elegendo prioridades, colabora com as orientações para a equipe de enfermagem proporcionando melhores condições de trabalho e elevando o padrão de atendimento institucional.

Diante do exposto, desperta o interesse o fato dessa Residência ser referência na área de gerência de enfermagem e por ser o único no país, de acordo com informações do COFEN descrito no relatório da visita in loco para reconhecimento do curso, realizado em 2009.
Nesse contexto, torna-se necessário identificar as linhas de pesquisa que estão vinculadas às monografias produzidas durante os seis anos de existência da residência e quantificar os artigos científicos e trabalhos, além dos autores envolvidos.

Mediante ao exposto, o presente estudo tem por objetivo descrever e qualificar a produção científica elaborada na Residência de Gerência dos Serviços de Enfermagem do Hospital Universitário da Universidade Estadual de Londrina - PR, no período de 2006 a 2011.

\section{Método}

Trata-se de um estudo transversal, documental, descritivo, de abordagem quantitativa. Foram analisados 69 artigos científicos e 96 trabalhos apresentados em eventos científicos no período de 2006 a 2011.

Para obtenção dos dados foram utilizadas as seguintes estratégias: levantamento da produção científica elaborada pelos residentes de Gerência dos Serviços de Enfermagem de 2006 até 2011, leitura dos títulos e resumos dos artigos para posterior classificação nas linhas de pesquisa e construção de dois bancos de dados em planilha do Microsoft Excel versão 2010.

Os artigos publicados constam no site do departamento de Enfermagem da UEL: http:// www.uel.br/ccs/enfermagem/acessar.php/page38. html, mensalmente atualizado (UNIVERSIDADE ESTADUAL DE LONDRINA, 2014).

Com relação aos periódicos utilizouse a classificação Qualis da Coordenação de Aperfeiçoamento de Pessoal de Nível Superior CAPES, a qual ocorre por meio de um conjunto de procedimentos utilizados para estratificação da qualidade da produção intelectual dos programas de pós-graduação. Dessa forma, o Qualis afere a qualidade dos artigos e de outros tipos de produção, a partir da análise da qualidade dos veículos 
de divulgação, ou seja, periódicos científicos. A classificação destes periódicos é realizada pelas áreas de avaliação e passa por atualização anualmente. Esses veículos são enquadrados em estratos indicativos da qualidade - $\mathrm{A} 1$, o mais elevado; A2; B1; B2; B3; B4; B5; C - com peso zero (CAPES, 2014).

Os periódicos são classificados em A ou B quando atendem aos critérios de composição de corpo editorial e de consultores, além da periodicidade e regularidade em que aparecem, sendo diferenciados quando indexados no SCIELO, obtendo classificação A, ou quando são editados por sociedades científicas nacionais com significativa representatividade na área, conseguindo classificação B. Já os periódicos que não atendem aos critérios citados anteriormente recebem classificação C (CAPES, 2014).

Para efeito do estudo, os artigos foram categorizados quanto ao ano de elaboração, titulação dos autores (graduando, residente, docente, enfermeiro assistencial e funcionário do hospital universitário), classificação dos periódicos de acordo QUALIS/CAPES (A1, A2, B1, B2, B3, B4, B5), situação da divulgação do artigo (submetido, publicado, no prelo e resubmetido a outro periódico após recusa na $1^{\mathrm{a}}$ submissão) e linhas de pesquisa a qual pertence (formação de recursos humanos em enfermagem, gestão de serviços de enfermagem, saúde do trabalhador, psicodinâmica do trabalho em saúde, gerenciamento de risco, qualidade da assistência de enfermagem e o cuidado de enfermagem nas fases do ciclo vital e nos diversos níveis assistenciais).

Já os trabalhos apresentados em eventos científicos foram classificados quanto à(o) abrangência do evento em que foi apresentado (local, regional e nacional), modalidade de apresentação (oral ou pôster), ano de apresentação e premiação (ordem de classificação e tipo do prêmio).

O projeto de pesquisa foi enviado ao Comitê de Ética em Pesquisa da Universidade Estadual de Londrina e registrado no Sistema Nacional de Informação sobre Ética em Pesquisa envolvendo Seres Humanos com CAAE $n^{\circ}$ 0246.0.268.000-11, sendo aprovado, com parecer 297/2011.

\section{Resultados}

Para melhor compreensão do quantitativo de artigos científicos produzidos por turma de residentes, realizou-se a distribuição dos mesmos conforme a situação de sua divulgação para a comunidade científica, conforme apresentado na Tabela 1.

Tabela 1 - Distribuição de artigos científicos elaborados por residentes em Gerência dos Serviços de Enfermagem do Hospital Universitário de Londrina que foram submetidos, publicados, encontram-se no prelo, resubmetidos a outro periódico e que aguardam nova submissão. Londrina, PR, 2011.

\begin{tabular}{lccccccc}
\hline \multicolumn{1}{c}{ Artigos } & \multicolumn{7}{c}{ Turmas } \\
\cline { 2 - 8 } & $\mathbf{2 0 0 6}$ & $\mathbf{2 0 0 7}$ & $\mathbf{2 0 0 8}$ & $\mathbf{2 0 0 9}$ & $\mathbf{2 0 1 0}$ & $\mathbf{2 0 1 1}$ & Total \\
\hline Submetidos & - & 01 & 03 & 06 & 09 & 03 & 22 \\
Publicados & 01 & 07 & 11 & 11 & - & - & 30 \\
$\begin{array}{l}\text { Prelo } \\
\text { Resubmetidos a outro }\end{array}$ & - & - & - & 01 & 02 & - & 03 \\
periódico & - & 01 & 01 & 05 & 03 & - & 10 \\
Aguardam nova submissão & - & 01 & - & 03 & - & - & 04 \\
\hline Total & 01 & 10 & 15 & 26 & 14 & 03 & 69 \\
\hline
\end{tabular}

Fonte: Universidade Estadual de Londrina, 2011. 
Os dados da Tabela 1 demonstram que as turmas que iniciaram suas atividades na residência nos anos de 2008 e 2009 possuem o maior número de artigos publicados, correspondendo a onze $(15,9 \%)$, cada. Além disso, é possível observar que os egressos de 2009 apresentam cinco artigos (7,2\%) resubmetidos a periódicos e um artigo $(1,4 \%)$ aceito para publicação, assim como a turma de 2010 possui dois artigos $(2,9 \%)$ no Prelo.

Posteriormente, aparecem a turma de 2007, com sete $(10,1 \%)$ artigos publicados, e a turma de 2010 , com nove $(13,0 \%)$ artigos submetidos.

$\mathrm{Na}$ Tabela 2 encontra-se a classificação no sistema Qualis/CAPES dos periódicos aos quais foram submetidos ou publicados os artigos das residentes em Gerência.

Tabela 2 - Classificação Qualis/CAPES dos periódicos nos quais os artigos elaborados por residentes em Gerência dos Serviços de Enfermagem do Hospital Universitário de Londrina foram submetidos, publicados, encontram-se no prelo e resubmetidos. Londrina, PR, 2011.

\begin{tabular}{lccccccc}
\hline Classificação & \multicolumn{7}{c}{ Turmas } \\
\cline { 2 - 9 } Qualis/CAPES & $\mathbf{2 0 0 6}$ & $\mathbf{2 0 0 7}$ & $\mathbf{2 0 0 8}$ & $\mathbf{2 0 0 9}$ & $\mathbf{2 0 1 0}$ & $\mathbf{2 0 1 1}$ & Total \\
\hline A1 & - & - & - & - & - & 01 & 01 \\
A2 & - & 01 & 02 & 04 & 04 & - & 11 \\
B1 & 01 & 03 & 07 & 08 & 08 & 02 & 29 \\
B2 & - & 01 & 04 & 03 & 02 & - & 10 \\
B3 & - & 04 & 02 & 04 & - & - & 10 \\
B4 & - & - & - & 02 & - & - & 02 \\
B5 & - & - & - & 02 & - & - & 02 \\
\hline Total & 01 & 09 & 15 & 23 & 14 & 03 & 65 \\
\hline
\end{tabular}

Fonte: Universidade Estadual de Londrina, 2011.

De acordo com dados apresentados na Tabela 2 as turmas de 2009 e 2010 se destacam por possuirem quatro $(6,1 \%)$ produções encaminhadas a periódicos classificados como A2, cada, e a turma de 2011 é a única que possui uma $(1,5 \%)$ submissão a um periódico A1. Os egressos de 2009 também se destacam por ser a equipe que possui maior quantidade de artigos elaborados $(35,4 \%)$.

Outro dado interessante é o predomínio, com 44,6\% (29), de artigos científicos enviados e/ou publicados em periódicos B1, seguido por $16,9 \%$
(11) submetidos e/ou publicados em veículos com Qualis A2.

Ressalta-se o fato da quantidade de artigos apresentados nas Tabelas 1 e 2 não coincidirem. Isso ocorre porque na segunda tabela foram desconsiderados aqueles que aguardam resubmissão a outro periódico após recusa na $1^{\mathrm{a}}$ submissão.

A Tabela 3 apresenta os artigos elaborados conforme as linhas de pesquisa desenvolvidas por docentes que atuam na residência em Gerência dos Serviços de Enfermagem. 
Tabela 3 - Distribuição de artigos científicos de acordo com as linhas de pesquisa desenvolvidas na Residência em Gerência dos Serviços de Enfermagem do Hospital Universitário de Londrina. Londrina, PR, 2011.

\begin{tabular}{cccccccc}
\hline \multirow{2}{*}{ Turma } & \multicolumn{7}{c}{ Linhas de pesquisa } \\
\cline { 2 - 8 } & $\begin{array}{c}\text { Formação } \\
\text { RH Enf. }\end{array}$ & $\begin{array}{c}\text { Gestão de } \\
\text { Serv. Enf. }\end{array}$ & $\begin{array}{c}\text { Saúde } \\
\text { Trabalhador }\end{array}$ & $\begin{array}{c}\text { Psicod. Trab. } \\
\text { em Saúde }\end{array}$ & $\begin{array}{c}\text { Gerenc. } \\
\text { Risco }\end{array}$ & $\begin{array}{c}\text { Qualidade } \\
\text { Ass. Enf. }\end{array}$ & $\begin{array}{c}\text { Cuidado/Ciclos } \\
\text { da vida }\end{array}$ \\
\hline 2006 & - & 01 & - & - & - & - & - \\
2007 & 02 & - & 05 & - & 01 & 02 & - \\
2008 & 01 & - & 09 & - & 02 & 02 & 01 \\
2009 & 01 & 06 & 05 & - & 02 & 10 & 02 \\
2010 & 01 & 04 & 04 & 02 & 02 & 1 & - \\
2011 & 01 & 02 & - & - & - & - & - \\
\hline Total & 06 & 13 & 23 & 02 & 07 & 15 & 03 \\
\hline
\end{tabular}

Fonte: Universidade Estadual de Londrina, 2011.

A Tabela 3 apresenta a prevalência de artigos voltados para a temática da Saúde do Trabalhador $(33,3 \%)$, sendo o segundo e terceiro lugares ocupados por Qualidade da Assistência de Enfermagem e Gestão de Serviços de Enfermagem, com $21,7 \%$ e $18,8 \%$ respectivamente.

Dos textos analisados, três $(4,3 \%)$ foram classificados na linha de pesquisa do cuidado de enfermagem nas fases do ciclo vital e nos diversos níveis assistenciais, pois os docentes que atuam nessa residência também desenvolvem atividades assistenciais em projetos de extensão e pesquisa.

$\mathrm{Na}$ Tabela 4 são descritos os trabalhos apresentados em eventos científicos.

Tabela 4 - Distribuição dos trabalhos elaborados por residentes em Gerência dos Serviços de Enfermagem do Hospital Universitário de Londrina, PR no período de 2006 a 2011, segundo modalidade, ano de apresentação e premiação. Londrina, PR, 2011.

\begin{tabular}{lcccccccc}
\hline \multirow{2}{*}{ Modalidade } & \multicolumn{7}{c}{ Ano de apresentação } \\
\cline { 2 - 9 } & & $\mathbf{2 0 0 6}$ & $\mathbf{2 0 0 7}$ & $\mathbf{2 0 0 8}$ & $\mathbf{2 0 0 9}$ & $\mathbf{2 0 1 0}$ & $\mathbf{2 0 1 1}$ & Total \\
\hline Pôster & 01 & 03 & 10 & 25 & 24 & 27 & 90 \\
\multirow{2}{*}{ Oral } & Premiados & - & - & 01 & 01 & 05 & 01 & \\
& & - & 02 & 01 & 02 & 01 & - & 06 \\
& Premiados & - & - & - & - & 01 & - & \\
\hline Total & & 01 & 05 & 11 & 27 & 25 & 27 & 96 \\
\hline
\end{tabular}

Fonte: Universidade Estadual de Londrina, 2011.

Verifica-se na Tabela 4 o predomínio das 8,9\% foram premiados. apresentações na modalidade pôster, com 93,7\% dos trabalhos apresentados desta forma. Destes, apenas uma (16,7\%) foi premiada. 
Tabela 5 - Distribuição dos trabalhos elaborados por residentes em Gerência dos Serviços de Enfermagem do Hospital Universitário de Londrina, conforme abrangência do evento de pesquisa. Londrina, PR, 2011.

\begin{tabular}{lccc}
\hline \multirow{2}{*}{ Ano } & \multicolumn{3}{c}{ Abrangência do evento de pesquisa } \\
\cline { 2 - 4 } & Regional & Nacional & Internacional \\
\hline 2006 & - & 01 & - \\
2007 & 02 & 03 & - \\
2008 & 06 & 05 & - \\
2009 & 03 & 06 & 18 \\
2010 & 02 & 23 & - \\
2011 & 02 & 22 & 3 \\
\hline Total & 15 & 60 & 21 \\
\hline
\end{tabular}

Fonte: Universidade Estadual de Londrina, 2011.

Observa-se que 62,5\% dos trabalhos foram apresentados em evento nacional, seguido por $21,9 \%$ em eventos internacionais e $15,6 \%$ em regionais.

\section{Discussão}

As pesquisas desenvolvidas e os trabalhos apresentados em eventos científicos demonstram que a pós-graduação na modalidade de Residência colabora para a qualificação de todos os profissionais envolvidos no desenvolvimento do curso, uma vez que envolve a participação dos enfermeiros assistenciais, residentes e docentes na construção do conhecimento pra área da enfermagem (LOPES; MOURA, 2004).

Com o maior número de enfermeiros assistenciais envolvidos com a pesquisa e o ensino no curso de Residência, houve também um aumento das publicações na área de enfermagem em comparação a outras áreas. Assim, infere-se que a Residência em enfermagem proporciona que os enfermeiros dos campos de estágio, que atuavam apenas na pratica assistencial, vinculem-se à academia e no desenvolvimento de pesquisas cientificas.
Todos os artigos apresentaram co-autores, variando de um a cinco, porém a maioria dos artigos (33,3\%) possuía quatro co-autores, seguido por 29,0\% que apresentavam três co-autores. A partir deste fato, considera-se que existe uma tendência para a realização de pesquisas em grupos, o que é importante para o desenvolvimento da produção científica da enfermagem (COSTA; CARVALHO, 2001).

Verificou-se a participação de alunos da graduação na realização dos estudos, o que ressalta a importância da iniciação científica no sentido de possibilitar que o discente tenha noções teóricas e metodológicas de pesquisa. A participação dos graduandos em projetos de pesquisa enriquece a formação escolar do aluno, fornece-lhe o alicerce para a continuidade dos estudos nos programas de pós-graduação, principalmente porque a UEL dispõe de Residência e Mestrado em Enfermagem, o que atua como mais um estímulo à capacitação profissional dos recém-formados (OLIVEIRA; HADDAD; BORTOLETTO, 2010).

Além de alunos da graduação envolvidos nas pesquisas, alguns artigos apresentaram como coautores docentes de outras instituições e áreas, 
demonstrando vínculo entre os programas de pósgraduação e a constante estímulo dos docentes que atuam na Residência/HUL/UEL em formar profissionais críticos, com visão ampliada do funcionamento hospitalar e adequada às necessidades do mercado de trabalho.

Observou-se que alguns servidores técnicos administrativos e administradores, foram envolvidos na produção científica da Residência em Gerência dos Serviços de Enfermagem, assessorando a coleta dos dados e discussão dos resultados.

Das seis linhas de pesquisa, a que mais apresentou artigos publicados foi a temática da Saúde do Trabalhador (33,3\%), seguido por Qualidade da Assistência de Enfermagem e Gestão de Serviços de Enfermagem, 21,7\% e 18,8\%, respectivamente. Verifica-se um maior engajamento dos profissionais de enfermagem nas discussões referentes as políticas de saúde e participação mais efetiva em órgãos de classe ou decisórios, além de uma maior preocupação em analisar a enfermagem enquanto prática social. $O$ profissional de enfermagem necessita articular os diferentes padrões do conhecimento para atingir a verdadeira integração entre pesquisa, prática e teoria, por meio de distintas metodologias, competências e fontes do conhecimento válidos e apropriados para seus propósitos (PERSEGONA et al., 2009).

Sobre os artigos e trabalhos produzidos, sua divulgação é importante, pois permite que outros profissionais tenham a oportunidade de conhecer, avaliar e questionar problemas que eventualmente possam surgir sobre as questões estudadas e solucionar conflitos pré-existentes no campo assistencial/ administrativo.

Dos 69 artigos elaborados, 44,6\% (29) foram submetidos e/ou publicados em periódicos B1, que segundo o Qualis/CAPES para a Enfermagem é uma classificação considerável.

Os trabalhos desenvolvidos sob a forma de pôster destacaram-se em relação à apresentação oral, assim como a participação em eventos nacionais.
Os autores dos trabalhos premiados $(9,4 \%)$, além do certificado, também receberam brindes em equipamentos utilizados pela equipe de enfermagem, prêmio em dinheiro ou direito à publicação em revista científica.

Durante a realização dessa pesquisa observou-se a apresentação de um mesmo trabalho em mais de um evento, sendo seis apresentados em três diferentes eventos e um, em quatro. Com esse conhecimento a partir de 2011 definiu-se que cada trabalho poderia ser apresentado em somente dois eventos científicos: um nacional e outro local.

\section{Considerações Finais}

Este estudo permitiu avaliar a produção científica da Residência em Gerência dos Serviços de Enfermagem. Constatou-se a elaboração de 69 artigos e 96 trabalhos científicos, apresentados em eventos regionais, nacionais e internacionais, pelas egressas e residentes.

A principal linha de pesquisa seguida pelos artigos foi Saúde do Trabalhador (33,3\%), seguido por Qualidade da Assistência de Enfermagem e Gestão de Serviços de Enfermagem (21,7\% e 18,8\%, respectivamente) e desses artigos $44,6 \%$ foram submetidos a periódicos B1.

Identificou-se o envolvimento de enfermeiros de serviços e graduandos no desenvolvimento das pesquisas, o que contribui para a qualificação do profissional que ingressará no mercado de trabalho e dos que já estão atuando e poderão aplicar os resultados das pesquisas no cotidiano da assistência de enfermagem.

Com relação aos trabalhos apresentados em eventos científicos, nove foram premiados e a maioria foi apresentada sob a forma de pôster, fato este que demonstra a importância e qualidade dos estudos desenvolvidos pelos profissionais que compõe essa Residência.

Assim, a partir do investimento na qualificação dos enfermeiros, com a implantação da Residência, tem sido possível aperfeiçoar o perfil do profissional e conquistar novos espaços na equipe de saúde e no mundo do 
trabalho. Observa-se que $62,5 \%$ dos trabalhos foram apresentados em evento nacional, seguido por $21,9 \%$ em eventos internacionais e $15,6 \%$ em regionais (Tabela $5)$.

\section{Referências}

CONSELHO FEDERAL DE ENFERMAGEM. Resolução $n^{\circ} .259$ de 12 de agosto de 2001 (BR). Dispõe sobre os padrões mínimos para registro do Enfermeiro Especialista, na modalidade de Residência em Enfermagem e dá outras providências. 2001. Disponível em: <http://www.portal-cofen.com.br/ legislacao/resolucoes.htm>. Acesso em: 2 jun. 2011.

COORDENAÇÃO DE APERFEIÇOAMENTO DE PESSOAL DE NÍVEL SUPERIOR - CAPES. Qualis Periódicos. Disponível em: <http://www.capes.gov. br/avaliacao/qualis $>$. Acesso em: 7 jul. 2014.

COSTA, R. S.; CARVALHO, D. V. Análise da produção científica dos enfermeiros de Minas Gerais publicada em periódicos de Enfermagem. Revista Latino-Americana de Enfermagem, Ribeirão Preto, v. 9, n. 5, p. 19-25, 2001.

GUARIENTE, M. H. D; ZAGO, M. F.; SOUBHIA, Z.; HADDAD, M. C. L. Sentidos da pesquisa na prática profissional de enfermeiras assistenciais. Revista Brasileira de Enfermagem, Brasília, v. 63, n. 4, 2010.

LOPES, G. T.; MOURA, C. F. S. O impacto da residência de enfermagem na reconfiguração do perfil do enfermeiro assistencial. Escola Anna Nery Revista de Enfermagem, Rio de Janeiro, v. 8, n. 4, p. 39-45, 2004.

MUNARI, D. B.; CHAVES, L. D. P.; PEDUZZI, M.; LAUS, A. M.; FUGULIN, F. M. T.; RIBEIRO, L. C. M.; SCOCHI, C. G. S. Cenário das pesquisas na pósgraduação na área de enfermagem e gerenciamento no Brasil. Revista da Escola de Enfermagem da USP, São Paulo, v. 45, n. esp., 2011.

OLIVEIRA, T. C. S.; HADDAD, M. C. L.; BORTOLETTO, M. S. S. Produção científica de graduandos em Enfermagem publicada de 1995 a 2008. Revista Espaço para a Saúde, Londrina, v. 11, n. 1, p. 21-28, 2010.
PERSEGONA, K. R.; ROCHA, D. .L. B.; LENARDT, M. H.; ZAGONEL, I. P. S. O conhecimento político na atuação do enfermeiro. Escola Anna Nery Revista de Enfermagem, Rio de Janeiro, v. 13, n. 3, p. 645-650, 2009.

SANTOS, I.; CASTRO, C. B. Características pessoais e profissionais de enfermeiros com funções administrativas atuantes em um hospital universitário. Revista da Escola de Enfermagem da USP, São Paulo, v. 44, n. 1, p. 154-160, 2010.

SILVA, V.; HOLZMANN, A. P. F.; VERSIANI, C.; FIGUEIREDO, M. F. S.; LIMA, A. C. A.; VIEIRA, M. A; SENA, R. R. Análise dos trabalhos de conclusão de curso da graduação em enfermagem da UNIMONTES. Revista Eletrônica de Enfermagem, Goiania, v. 11, n. 1, p. 133-143, 2009.

UNIVERSIDADE ESTADUAL DE LONDRINA UEL. Departamento de Enfermagem. Residências em enfermagem. Disponível em: <http://www.uel.br/ccs/ enfermagem/acessar.php/res.html $>$. Acesso em: 7 jul. 2014.
Recebido em: 09 jul. 2014. Aceito em: 12 ago. 2014. 
